\title{
FEATURES OF SHORT-PERIOD VARIABILITY OF TOTAL ELECTRON CONTENT AT HIGH AND MIDDLE LATITUDES
}

\author{
A.S. Yasyukevich \\ Institute of Solar-Terrestrial Physics SB RAS, \\ Irkutsk, Russia,annpol@iszf.irk.ru
}

\begin{abstract}
The study presents the results of comparative analysis of features of a short-period (with periods of internal gravity waves) variability of total electron content (TEC) in the ionosphere at middle (Novosibirsk) and high (Norilsk) latitudes over a long period of time (2003-2020). The period analyzed makes it possible to estimate not only diurnal and seasonal variations in the variability, but also its changes within the solar activity cycle. The level of TEC variability is shown to experience pronounced seasonal variations with maxima in winter months. The difference between the level of variability in winter and summer is about two times for Novosibirsk and up to seven times for Norilsk. The variability features a distinct diurnal variation; however, the diurnal dependence at the mid- and high-latitude stations differs significantly. At high latitudes, the level of variability in the winter period strictly depends on solar activity. For the mid-latitude station, there is no clear dependence of variability level on solar activity; in the years of solar maximum, on the contrary, a slight decrease in the variability is observed. In summer, the level of variability at both middle and high latitudes
\end{abstract}

remains practically unchanged and does not depend on solar activity. The main features in the dynamics of variability are shown to be similar at stations located at other longitudes, except for the East American sector. The result obtained suggests that the short-period TEC variability at high latitudes is primarily related to changes in solar activity, but regular variations in the variability at midlatitudes are probably not associated with heliophysical activity. The observed increase in the level of short-period variability in the winter midlatitude ionosphere is assumed to be related to an increase in wave activity in the stratosphere.

Keywords: ionosphere; total electron content; GPS; ionospheric variability.

\section{INTRODUCTION}

Earth's upper atmosphere is a region affected by the Sun and the magnetosphere, as well as by underlying layers of the neutral atmosphere. The determining factor in the thermodynamic regime of the upper atmosphere is heliogeomagnetic activity. At the same time, studies indicate that the ionospheric variations observed can be caused by atmospheric waves of different space-time scales: acoustic, internal gravity (IGW), tidal, and planetary waves propagating from the lower and middle atmosphere [Forbes et al., 2000; Lastovicka, 2006]. Under certain conditions, IGW from sources located in the lower atmosphere can penetrate to great heights, causing wave disturbances in parameters of the upper mesosphere, thermosphere, and hence ionospheric plasma [Hocke, Schlegel, 1996], thereby ensuring the coupling between atmospheric layers. Using simulation results, Liu et al. [2013] have demonstrated that meteorological forcing can play a key role in the day-to-day variability of the ionosphere. The combination of these effects determines properties of the ionospheric disturbance in different ranges of periods.

Propagation of acoustic and internal gravity waves in the ionosphere causes short-period (with periods to
5-6 hrs) disturbances [Medvedev et al., 2013]. Properties of short-period electron density disturbance in the F2 layer of the ionosphere have been studied in [AraujoPradere et al., 2005; Altadil, 2007; Ratovsky et al., 2015]. The normalized standard deviation of a selected ionospheric parameter in a given interval was generally used as a criterion for assessing the disturbance [AraujoPradere et al., 2005; Altadil, 2007]. This approach was also applied when developing disturbance models [Mendillo et al., 2002]. Nesterov et al. [2017] have proposed a method of normalizing to the standard deviation, rather than to the mean value (as is traditionally done), in order to construct a relative disturbance index. This approach allows one to avoid the possible dependence of the relative disturbance on the seasonal diurnal variation in the value under study.

The level of ionospheric disturbance with IGW periods (from $10 \mathrm{~min}$ to several hours) was established to have a pronounced seasonal dependence at high and middle latitudes. Under quiet geomagnetic conditions, the lowest variability was found in the summer period; the maximum, in winter; and intermediate values of the disturbance were recorded during equinoxes. Ratovsky et al. [2015] also confirmed that the overall relative variability obtained from digisonde data in Irkutsk has a 
clear seasonal variation with a maximum in winter, a minimum in summer, and with intermediate values during equinoxes.

It was also revealed that the disturbance depends on local time (LT). For example, for the mid-latitude station Ebro it was shown that the greatest variability in the F-region is recorded at night time [Altadil, 2007].

Nonetheless, the question about the relationship between the disturbance level and solar activity remains open. For example, Ratovsky et al. [2015] have noted the absence of a clear dependence of the level of shortperiod electron density variability in Irkutsk on an increase in solar activity. The authors concluded that it remains unclear how to interpret the result since activity of gravity waves increases with solar activity [Medvedev et al., 2013]. Probably, the insufficiently long period of the study did not allow identifying the possible relationship.

This paper reports the results of comparative analysis of a short-period variability of the ionospheric total electron content (TEC) at middle and high latitudes. The period (2003-2020) under analysis includes almost two solar cycles, allowing us to estimate not only diurnal and seasonal variations in the short-period TEC variability, but also oscillations within the solar activity cycle.

\section{METHOD FOR ESTIMATING SHORT-PERIOD TEC VARIABILITY}

The study is based on TEC data obtained from measurements of dual-frequency GPS/GLONASS receivers of the international IGS network, located at close longitudes in mid-latitude (Novosibirsk, NVSK, $53^{\circ} \mathrm{N}, 83^{\circ} \mathrm{E}, 50^{\circ}$ Glat, $156^{\circ}$ Glon) and high-latitude (Norilsk, NRIL, $69^{\circ} \mathrm{N}, 88^{\circ} \mathrm{E}, 64^{\circ}$ Glat, $162^{\circ}$ Glon) regions. Corrected geomagnetic coordinates of the stations (Glat, Glon) were calculated according to the IGRF model for 2010 [https://omniweb.gsfc.nasa.gov/vitmo/cgm.html].

From phase and code measurements, series of vertical absolute TEC are calculated by the method described in [Yasyukevich et al., 2020b]. This method provides long-term series of non-negative values of absolute TEC, taking into account differential code biases. The time resolution of the TEC values obtained is 15 min. The estimates given in [Yasyukevich et al., 2020b] show that the deviation of the TEC values obtained by this method from the TEC values calculated by alternative methods (e.g., from global ionospheric maps) does not exceed 1-2.5 TECU. This corresponds to the discrepancy between TEC values according to maps from different laboratories.

To isolate short-period variations from the TEC series, we have applied the method described in [Ratovsky et al., 2015], used to analyze variations in the maximum electron density of the F2 layer from Irkutsk ionosonde data. The application of this method is also justified by the possibility of comparing results for TEC and $N_{\mathrm{m}} \mathrm{F} 2$. The method involves the following stages:
1. For each moment of LT, the normalized deviation of TEC from the 27-day median value is determined:

$$
\operatorname{VTEC}(L T)=\frac{T E C(L T)-\langle T E C(L T)\rangle_{27}}{\langle T E C(L T)\rangle_{27}} .
$$

2. To separate the contributions of long-period $\left(\triangle T E C(L T)_{\mathrm{LONG}}\right)$ and short-period $\left(\triangle T E C(L T)_{\mathrm{IGW}}\right)$ variations, the series are filtered with a running window of 6 hrs. Low-frequency TEC variations $\left(\triangle T E C_{\mathrm{LONG}}\right.$, with periods over $6 \mathrm{hrs}$ ) are related to day-to-day ionospheric variability, as well as to tidal variations with diurnal and semidiurnal components. For each time instance, the variability in IGW ranges is defined as

$$
\Delta T E C(L T)_{\mathrm{IGW}}=\Delta T E C(L T)-\Delta T E C(L T)_{\mathrm{LONG}} .
$$

Thus, given the time resolution of the initial TEC data, the range of periods of the disturbances obtained is $0.5-6$ hrs. Note that the time resolution of the initial data on vertical TEC does not allow us to estimate the contribution of the smallest-scale acoustic disturbances; therefore, in this paper, by the short-period disturbance is meant variations with periods close to IGW.

3 . To determine the variability coefficient, the standard deviation $\sigma$ of $\triangle T E C(L T)_{\mathrm{IGW}}$ at the given interval is calculated: for each day, three disturbance coefficients are found, with the standard deviation calculated separately only during the daytime or only at night, as well as throughout the days. To separate daytime and nighttime conditions, the time of passage of the solar terminator at an altitude of $100 \mathrm{~km}$ is used.

To obtain the diurnal seasonal dynamics of the variability for each moment of LT, the standard deviation $\sigma$ of TEC $(L T)_{\mathrm{IGW}}$ is calculated for all the years analyzed.

4. The purpose of this work is to study properties of TEC variability under quiet geomagnetic conditions. Therefore, sharp bursts exceeding the threshold of $2 \sigma$ from the 27-day moving median are excluded from the disturbance series. Such bursts can be linked either to geomagnetic disturbances or to errors in estimating TEC.

To identify seasonal variations in the dynamics of the variability coefficients, the series with a period of 27 days were smoothed.

\section{RESULTS}

Figure 1 presents series of coefficients of shortperiod TEC variability, calculated as $\sigma$ of $\triangle T E C(L T)_{\mathrm{IGW}}$ for one local day, as well as their values smoothed over a period of 27 days (gray curve) for Novosibirsk (NVSK, bottom) and Norilsk (NRIL, top) in 2005.

The coefficients smoothed over a period of 27 days reflect seasonal variations in variability. In addition, smoothed variability coefficients calculated separately only during local daytime (red curve) and only during local nighttime (blue curve) are plotted.

The level of variability is seen to experience significant regular diurnal and seasonal fluctuations. The smallest $T E C_{\mathrm{IGW}}$ values are recorded at both latitudes in summer months. This finding is in agreement with 

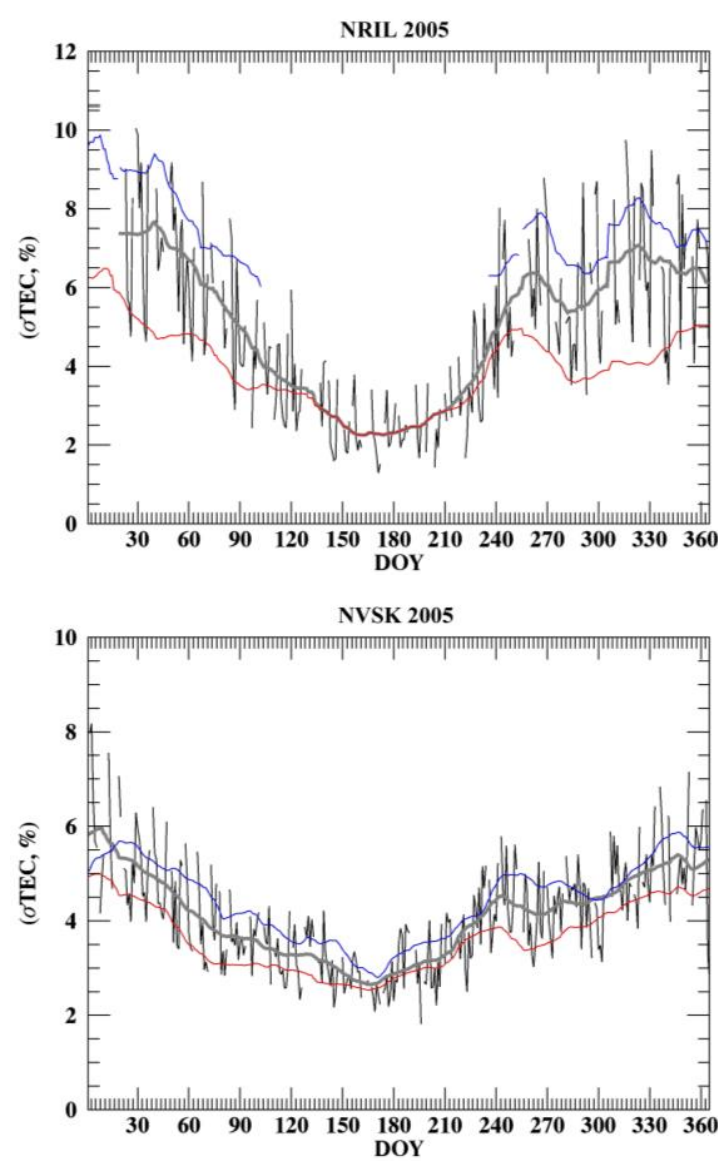

Figure 1. Series of coefficients of short-period TEC variability, as well as their values smoothed over a period of 27 days (gray curve) for Novosibirsk (NVSK, bottom) and Norilsk (NRIL, top) stations in 2005. Additionally, smoothed variability coefficients calculated only during local daytime (red curve) and only during local nighttime (blue curve) are plotted

previously obtained ones for electron density disturbance in the region of the ionospheric maximum.

Note that at the mid-latitude station NVSK the level of variability at nighttime is only slightly (1.2-1.3 times) higher than the level of disturbance in the daytime. At the same time, the difference between daytime and nighttime values decreases in summer months.

At the high-latitude station, the difference between daytime and nighttime variability is much more pronounced - on average, the nighttime disturbance may be more than twice as great as the daytime one. In summer months, the nighttime disturbance was not estimated because of the polar day during this period at an altitude of $100 \mathrm{~km}$ and higher.

Seasonal variations in the coefficient of short-period variability in different years are shown in Figure 2 for Norilsk (left) and Novosibirsk (right) for 2003-2020. The thick line indicates seasonal variations averaged over all the years of interest. In the top panel are seasonal variations for the disturbance calculated for a local day; in the middle panel, only for the daytime; and in the bottom panel, for the nighttime.

In the dynamics of variability, there is a pronounced seasonal variation with maxima in winter. This behavior is observed regularly from year to year. The difference between the level of disturbance in winter and summer is nearly two times for Novosibirsk and up to seven times for Norilsk. The seasonal differences are clearly seen both during nighttime and daytime, yet more pronounced at night.

Referring to Figure 2, the behavior of summer and winter TEC variability may differ significantly. Therefore, to assess the effect of solar activity on the level of short-period TEC variability for each year under study, average disturbance coefficients are calculated separately for summer and winter periods.

Figure 3 shows variations in the winter and summer average variability versus variations in solar activity (the $F 10.7$ index). In the top panel, the disturbance was calculated for the local day; in the middle panel, only for the daytime, in the bottom panel, only for the nighttime. Numbers in the panels denote the correlation coefficients between $F 10.7$ variations and the variability index in winter $\left(C_{\mathrm{w}}\right)$ and summer $\left(C_{\mathrm{s}}\right)$ periods respectively.

It is obvious that at the high-level station NRIL the level of disturbance in winter depends on solar activity; this dependence is more pronounced at night $\left(C_{\mathrm{w}}\right.$ is as high as 0.7 ). With decreasing solar activity, the disturbance decreases by $\sim 50 \%$. This implies that the intensity of the short-period TEC variability at high latitudes is determined by the level of solar activity.

For the mid-latitude station NVSK, such a dependence on the $F 10.7$ level is not observed. This is consistent with the results obtained by Ratovsky et al. [2015] for the electron density disturbance over Irkutsk. This result indicates that regular ionospheric disturbance variations in the IGW range at midlatitudes are likely unrelated to heliophysical activity. During years of high solar activity, on the contrary, there is a slight decrease in the disturbance level.

Note that the disturbance level in summer months at high latitudes remains practically unchanged $(\sim 2 \%)$, regardless of solar activity. There is also no significant correlation between $F 10.7$ and the variability index $\left(C_{\mathrm{s}} \sim 0.2\right)$. For the mid-latitude station in the summer period, there is a pronounced anticorrelation between the level of solar activity and disturbance $\left(C_{\mathrm{s}}\right.$ is over -0.8$)$. It is notable that in summer the average level of disturbance at NVSK appears to be even slightly higher than at NRIL.

A possible explanation for the decrease in the level of relative disturbance during years of solar maximum at midlatitudes may be the fact that with an increase in solar activity, photochemical processes begin to prevail over dynamic ones (wave disturbances) and ionospheric plasma becomes more resistant to the weak impact of wave disturbances. The weakening of photochemical processes at night can also explain the predominance of the nighttime disturbance over the daytime one at midlatitudes [Rishbeth, Mendillo, 2001; Altadil, 2007].

Figure 4 displays diurnal and seasonal variations of the short-period TEC variability in Norilsk and Novosibirsk for 2003-2020. To obtain these distributions for each moment of LT, the standard deviations of $\triangle T E C(L T)_{\mathrm{IGW}}$ were calculated for all the years under study.

At both stations, there is a pronounced diurnal and seasonal variation in the variability; however, the nature of this dependence is significantly different. 

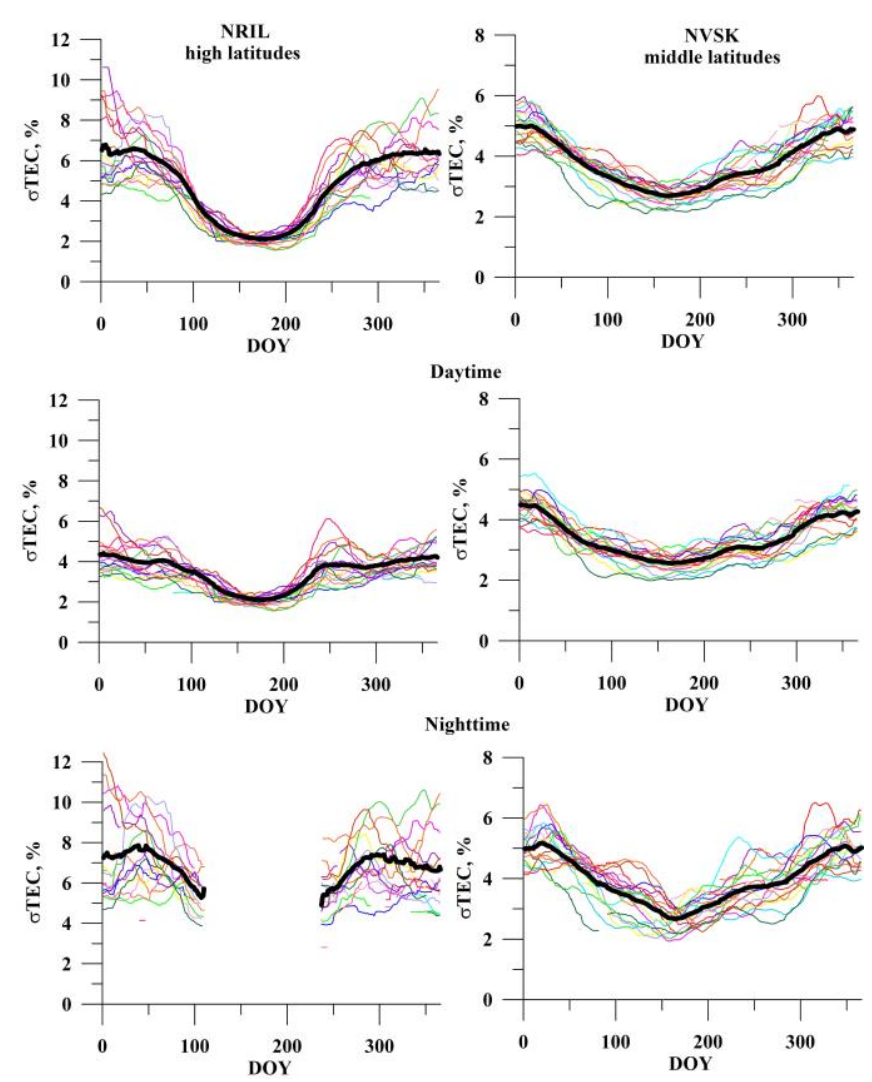

Figure 2. Seasonal variations in short-period TEC variability in different years for Norilsk (NRIL, left) and Novosibirsk (NVSK, right). The thick curve shows the variations obtained by averaging over all the years under study
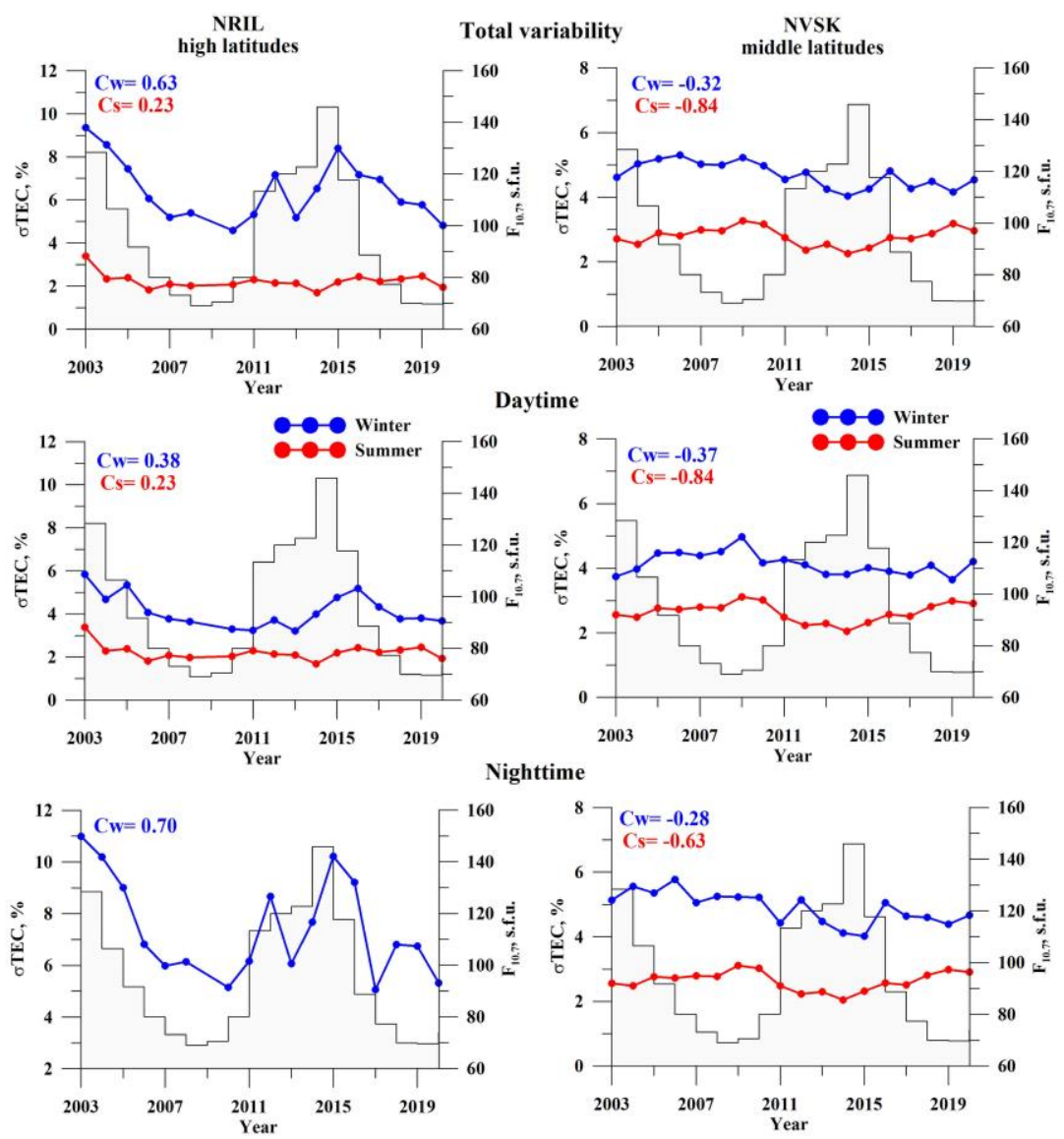

Figure 3. Dynamics of yearly averaged TEC variability in winter (black) and summer (gray) versus variations in annual average F10.7 (gray bars) in Norilsk (left) and Novosibirsk (right). Numbers in the panels indicate values of respective correlation coefficients 

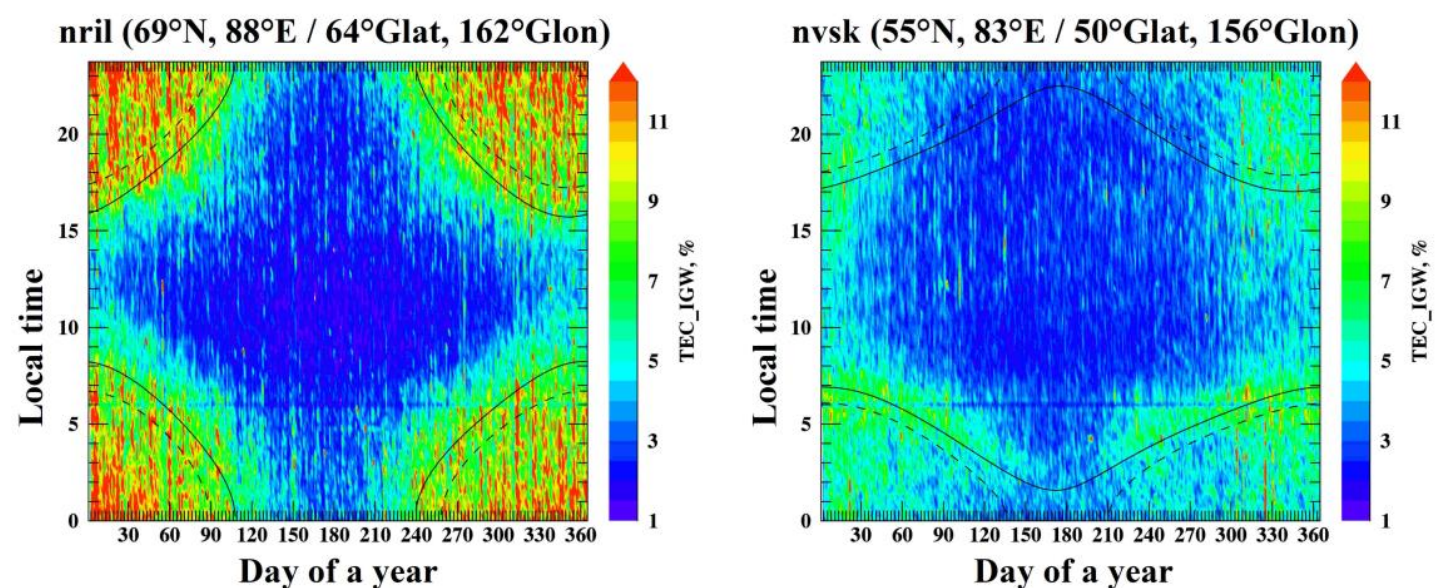

Figure 4. Diurnal seasonal variations in short-period TEC variability in Norilsk (left) and Novosibirsk (right). Lines indicate moments of passage of the solar terminator at heights of $100 \mathrm{~km}$ (solid line) and $300 \mathrm{~km}$ (dashed line)

At the high-latitude station NRIL, the disturbance increases significantly at night, immediately after the passage of the solar terminator (ST). This is probably linked to the manifestations of auroral activity. At night, the station gets closer to the auroral oval expanding toward lower latitudes, which exhibits the development of intense small-scale ionospheric disturbances. Thus, the shortperiod disturbance at the high-latitude station at night is determined mainly by processes in the auroral region.

At the mid-latitude station NVSK, there are two distinct peaks of disturbance near the moments of ST passage; in this case, the disturbance level near the dawn terminator is higher than that near the dusk one.

The motion of ST is a regular source of wave disturbances in the atmosphere and ionosphere, generating waves as well as causing instabilities and fluctuations to occur in ionospheric plasma [Francis, 1974]. Afraimovich et al. [2009a, b] found that the motion of ST is accompanied by generation of medium-scale disturbances of a special type in the ionosphere - wave packets manifested in the form of narrow-band TEC oscillations. A model of generation of magnetohydrodynamic plasma disturbances in the ionosphere - plasmasphere system has been proposed. Further studies showed [Edemskiy, Yasyukevich, 2011] that the TEC disturbances related to the ST passage can have a more complex structure, apparently being a superposition of ionospheric plasma oscillations caused by the passage of not only magnetohydrodynamic, but also gravity waves. Thus, the greatest contribution to the shortperiod TEC variability at midlatitudes is made by variations associated with the ST passage.

Note, however, that an increase in the level of disturbance is recorded not only near ST, but also throughout the nighttime. Moreover, during winter periods, the disturbance level remains increased throughout all days.

It is worth noting that the results obtained can be applied to stations located near the longitudinal region under study since the discrepancy between geomagnetic and geographic latitudes varies greatly depending on longitude. In this regard, diurnal and seasonal distributions of the variability at close geo- graphic latitudes, but in other longitudinal sectors characterized by different deviations between geomagnetic and geographic coordinates are examined. Figure 5 shows distributions for 2010-2020, similar to those depicted in Figure 4, for the pairs of stations located in the European (LAMA, SODA), West American (FAIR, HOLB), and East American (BAKE, DUBO) sectors.

It is obvious that the dynamics of variability as a whole has a similar character in different longitudinal sectors. The greatest differences are observed for stations in the East American sector, where stations at similar geographic longitudes are located closest to the geomagnetic pole. Thus, the variability level during the nighttime at BAKE is much higher than at other highlatitude stations considered. At the mid-latitude station DUBO, the variability level increases at night (immediately after the passage of ST) not only in winter, but also in summer. Nonetheless, based on the geomagnetic latitude of this station, it should be referred to as highlatitude rather than mid-latitude. Obviously, the main conclusions about the variability behavior drawn in this work should be applied to this longitudinal region with caution, always taking into account the geomagnetic latitude of a station. The above results are valid for other longitudinal sectors considered.

\section{DISCUSSION}

The regularly observed considerable seasonal variations in TEC variability at midlatitudes with winter maximum and summer minimum do not correlate with variations in solar activity. A source of this variability may be processes in the underlying atmosphere.

The following factors are considered as causes of the seasonal differences in the level of ionospheric IGW variability: seasonal variations in the photochemical regime [Araujo-Pradere et al., 2005] and changes associated with meteorological activity [Lastovicka, 2006; Altadill, 2007].

One of the IGW sources in the winter polar stratosphere and the lower mesosphere is the circumpolar vortex [Wu, Waters, 1996; Whiteway et al., 1997]. The vortex is a large-scale cyclonic circulation cell formed in the cold air mass over the polar region and covering 

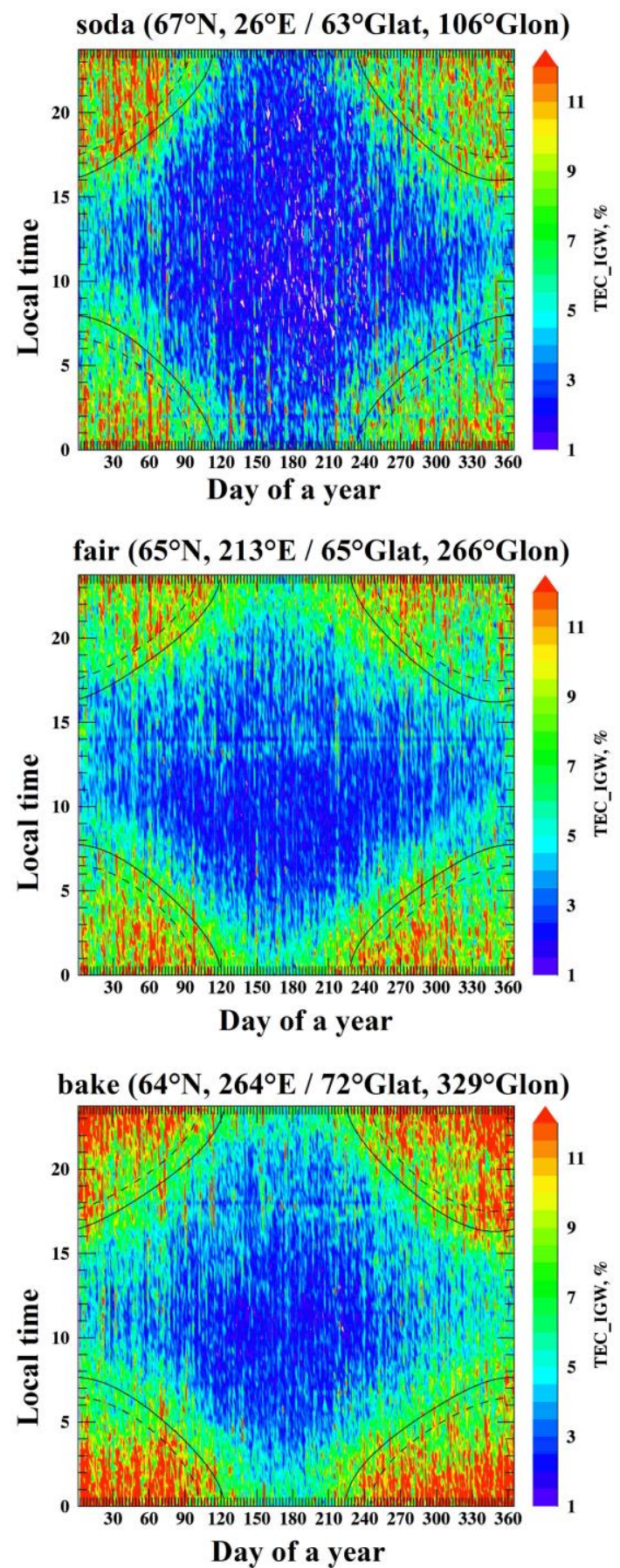

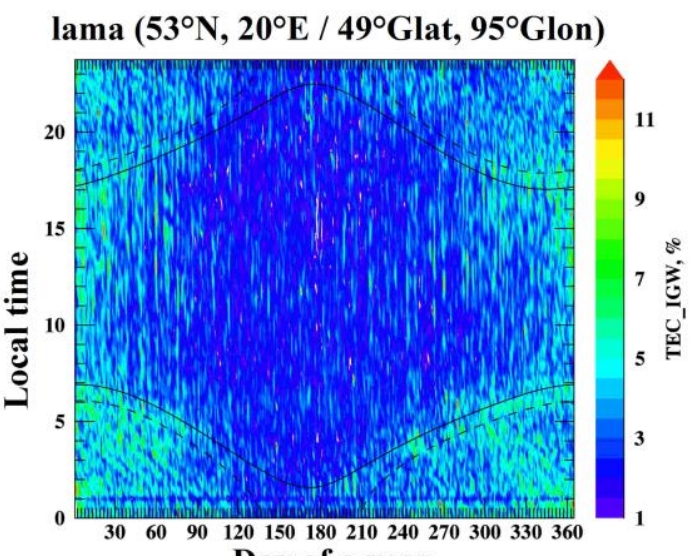

Day of a year
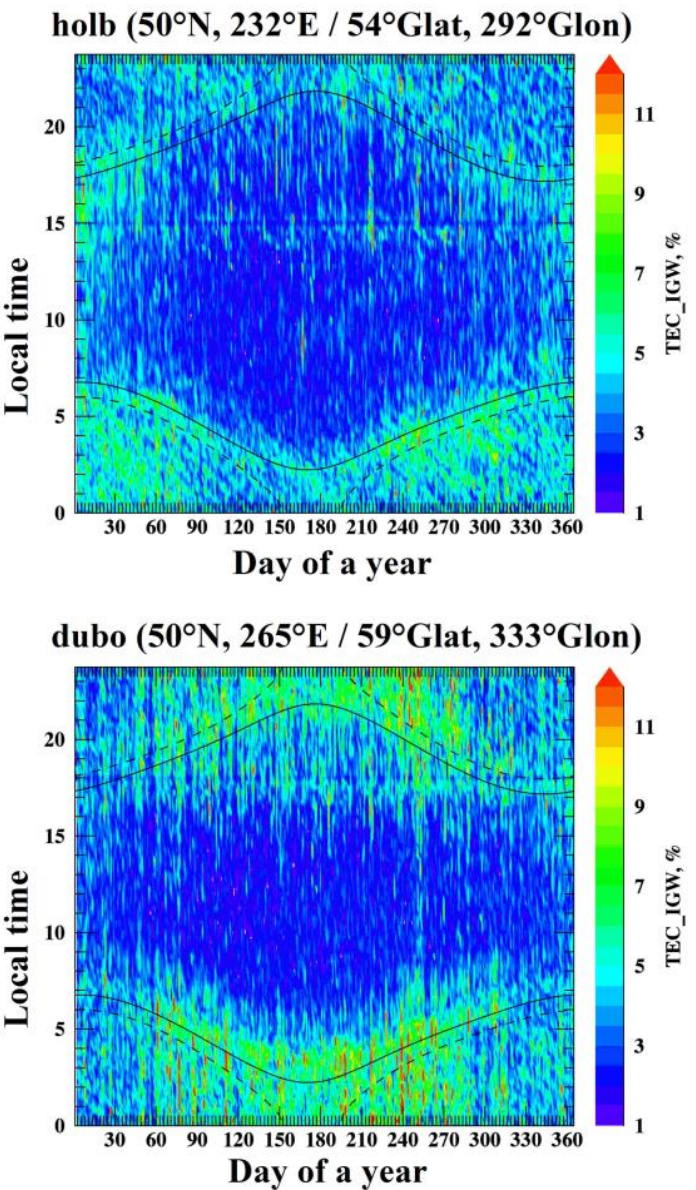

Figure 5. Diurnal and seasonal variations in short-period TEC variability in the European (top), West American (middle), and East American (bottom) sectors at high (left) and middle (right) latitudes. Station names and their coordinates are in the corresponding panels. Lines indicate moments of passage of the solar terminator at heights of $100 \mathrm{~km}$ (solid line) and $300 \mathrm{~km}$ (dashed line)

the upper troposphere and the stratosphere. The circumpolar vortex is followed by the development of a jet stream - a narrow, clearly limited atmospheric gas stream that features high velocities (up to $100 \mathrm{~m} / \mathrm{s}$ and higher) and large vertical and horizontal wind shears. It has been found that shear instabilities in a jet stream lead to generation of IGW-scale atmospheric waves at stratospheric heights (with periods from tens of minutes to several hours) [Shpynev et al., 2015]. Shpynev et al. [2019] have shown that up to 10-15\% of the total energy of jet stream can be spent on IGW generation. Frissell et al. [2016] concluded that there is no clear correlation between the level of variability of the mid-latitude ionosphere and the geomagnetic activity indices ( $A E$ and $S Y M-H)$; at the same time, the correlation with the dynamics of the circumpolar vortex is significant. The relationship between the intensification of the winter ionospheric disturbance and the dynamics of the stratospheric jet stream over Eurasia was also observed in [Chernigovskaya et al., 2018]. Yasyukevich et al. [2020a] have revealed that there is a clear correlation between the indices of short-period variability in the stratosphere, ionosphere, and at the height of the mesopause. 
Thus, the increase in the level of short-period variability in the mid-latitude ionosphere may be related to meteorological factors, namely, to an increase in wave activity in the stratosphere, associated with the development of the winter circumpolar vortex.

\section{CONCLUSION}

The paper has analyzed properties of the shortperiod TEC variability in the ionosphere at middle and high latitudes. The period of interest includes almost two solar cycles (2003-2020), which made it possible to estimate not only diurnal and seasonal variations in the disturbance, but also changes in its level depending on solar activity.

It has been shown that in the disturbance dynamics there is a pronounced seasonal variation with winter maxima. The difference between the level of variability in winter and summer is about two times for Novosibirsk and up to seven times for Norilsk. The seasonal differences are clearly seen both during nighttime and daytime, yet more pronounced at night.

The variability dynamics features a pronounced diurnal variation; however, the nature of the diurnal dependence at mid- and high-latitude stations differs significantly. At the high-latitude station, the disturbance increases significantly at night, immediately after the passage of the solar terminator, which is likely associated with manifestations of auroral activity intensifying at night. At the mid-latitude station, in the diurnal variation of the variability there are two pronounced peaks near the time of passage of solar terminators. Increased values of the disturbance level are also recorded in winter periods throughout the days.

At the high-latitude station NRIL, the variability level in winter strictly depends on solar activity, this dependence being more pronounced at night. From solar maximum to minimum, the variability level in winter decreases by more than $50 \%$. For the mid-latitude station NVSK, there is no clear dependence of the disturbance on solar activity; during years of solar maximum, on the contrary, a slight decrease in the disturbance level is observed. The result obtained suggests that the short-period TEC variability at high latitudes during the nighttime is associated primarily with changes in the level of solar activity; however, regular seasonal variations in the disturbance at midlatitudes are probably unrelated to heliophysical activity.

In summer, the variability level at high latitudes remains practically unchanged $(\sim 2 \%)$, regardless of solar activity. At the mid-latitude station in the summer period, there is a pronounced anticorrelation between the level of solar activity and TEC variability.

We assume that this increase in the level of shortperiod variability in the mid-latitude ionosphere may be associated with meteorological factors, namely, with an increase in wave activity in the stratosphere, related to the development of the winter circumpolar vortex.

These findings may be of practical value for the realtime prediction of the state of a radio channel and the deterioration in the operation of radio engineering systems, as well as may be used for developing and testing dynamic models of the ionosphere, depending on various factors. Understanding properties of the regular ionospheric variability is also valuable in studying the effects of various non-stationary events on the ionosphere.

This work was financially supported by the Russian Science Foundation (Grant No. 20-77-00070).

\section{REFERENCES}

Afraimovich E.L., Edemskiy I.K., Leonovich A.S., Leonovich L.A., Voeykov S.V., Yasyukevich Y.V. MHD nature of night-time MSTIDs excited by the solar terminator. Geophys. Res. Lett. 2009a, vol. 36, L15106. DOI: 10.1029/ 2009 GL039803.

Afraimovich E.L., Edemskiy I.K., Voeykov S.V., Yasukevich Yu.V., Zhivetiev I.V. Spatio-temporal structure of the wave packets generated by the solar terminator. Adv. Space Res. 2009b, vol. 44, pp. 824-835. DOI: 10.1016/j.asr.2009.05.017.

Altadill D. Time/altitude electron density variability above Ebro, Spain. Adv. Space Res. 2007, vol. 39, pp. 962969. DOI: 10.1016/j.asr.2006.05.031.

Araujo-Pradere E.A., Fuller-Rowell T.J., Codrescu M.V., Bilitza D. Characteristics of the ionospheric variability as a function of season latitude local time and geomagnetic activity. Radio Sci. 2005, vol. 40, RS5009. DOI: 10.1029/2004RS003179.

Chernigovskaya M.A., Shpynev B.G., Ratovsky K.G, et al. Ionospheric response to winter stratosphere/lower mesosphere jet stream in the Northern Hemisphere as derived from vertical radio sounding data. J. Atmos. Solar-Terr. Phys. 2018, vol. 180, pp. 126-136. DOI: 10.1016/j.jastp.2017.08.033.

Edemskiy I.K., Yasyukevich Y.V. Duration of wave disturbances generated by solar terminator in magneto-conjugate areas. Proc. XXX $X^{\text {th }}$ URSI General Assembly and Scientific Symposium. Istanbul, Turkey, 2011, pp. 1-4. DOI: 10.1109/ URSIGASS.2011.6051003.

Forbes J.M., Palo S.E., Zhang, X. Variability of the ionosphere. J. Atmos. Solar-Terr. Phys. 2000, vol. 62, iss. 8, pp. 685-693. DOI: 10.1016/S1364-6826(00)00029-8.

Francis S.H. A theory of medium-scale traveling ionospheric disturbances. J. Geophys. Res. 1974, vol. 79, iss. 34, pp. 5245-5260. DOI: 10.1029/JA079i034p05245.

Frissell N.A., Baker J.B.H., Ruohoniemi J.M., Greenwald R.A., Gerrard A.J., Miller E.S., West M.L. Sources and characteristics of medium-scale traveling ionospheric disturbances observed by high-frequency radars in the North American sector. J. Geophys. Res. 2016, vol. 121, pp. 37223739. DOI: 10.1002/2015JA022168.

Hocke K., Schlegel K. A review of atmospheric gravity waves and travelling ionospheric disturbances: 1982-1995. Ann. Geophys. 1996, vol. 14, pp. 917-940. DOI: 10.1007/ s00585-996-0917-6.

Lastovicka J. Forcing of the ionosphere by waves from below. J. Atmos. Solar-Terr. Phys. 2006, vol. 68, pp. 479-497. DOI: 10.1016/j.jastp.2005.01.018.

Liu H.-L., Yudin V.A., Roble R.G. Day-to-day ionospheric variability due to lower atmosphere perturbations. Geophys. Res. Lett. 2013, vol. 40, pp. 665-670. DOI: 10.1002/grl.50125.

Medvedev A.V., Ratovsky K.G., Tolstikov M.V., Alsatkin S.S., Scherbakov A.A. Studying of the spatialtemporal structure of wavelike ionospheric disturbances on the base of Irkutsk incoherent scatter radar and digisonde data. J. Atmos. Solar-Terr. Phys. 2013, vol. 105, pp. 350357. DOI: 10.1016/j.jastp.2013.09.001. 
Mendillo M., Rishbeth H., Roble R.G., Wroten J. Modelling F2-layer seasonal trends and day-to-day variability driven by coupling with the lower atmosphere. J. Atmos. Solar-Terr. Phys. 2002, vol. 64, pp. 1911-1931. DOI: 10.1016/ S1364-6826(02)00193-1.

Nesterov I.A., Andreeva E.S., Padokhin A.M., Tumanova Yu.S., Nazarenko M.O. Ionospheric perturbation indices based on the low- and high-orbiting satellite radio tomography data. GPS Solut. 2017, vol. 21, pp. 1679-1694. DOI: 10.1007/s10291-017-0646-1.

Ratovsky K.G., Medvedev A.V., Tolstikov M.V. Diurnal, seasonal and solar activity pattern of ionospheric variability from Irkutsk Digisonde data. Adv. Space Res. 2015, vol. 55, pp. 2041-2047. DOI: 10.1016/j.asr.2014.08.001.

Rishbeth H., Mendillo M. Patterns of F2-layer variability. J. Atmos. Solar-Terr. Phys. 2001, vol. 63, pp. 1661-1680. DOI: $10.1016 / \mathrm{S} 1364-6826(01) 00036-0$.

Shpynev B.G., Churilov, S.M., Chernigovskaya M.A. Generation of waves by jet-stream instabilities in winter polar stratosphere/mesosphere. J. Atmos. Solar-Terr. Phys. 2015 , vol. 136(B), pp. 201-215. DOI: 10.1016/j.jastp.2015.07.005.

Shpynev B.G., Khabituev D.S., Chernigovskaya M.A., Zorkal'tseva O.S. Role of winter jet stream in the middle atmosphere energy balance. J. Atmos. Solar-Terr. Phys. 2019 , vol. 188, pp. 1-10. DOI: 10.1016/j.jastp.2019.03.008.
Whiteway J.A., Duck T.J., Donovan D.P., Bird J.C., Pal S.R., Carswell A.I. Measurements of gravity wave activity within and around the Arctic stratospheric vortex. Geophys. Res. Lett. 1997, vol. 24, iss. 11, pp. 1387-1390. DOI: 10.1029/97GL01322.

Wu D.L., Waters J.W. Satellite observations of atmospheric variances: A possible indication of gravity waves. Geophys. Res. Lett. 1996, vol. 23, iss. 11, 24, pp. 36313634. DOI: 10.1029/96GL02907.

Yasyukevich A., Medvedeva I., Sivtseva V., Cher... govskaya M., Ammosov P., Gavrilyeva G. Strong Interrelation between the Short-Term Variability in the Ionosphere, Upper Mesosphere, and Winter Polar Stratosphere. Remote Sens. 2020a, vol. 12, 1588. DOI: 10.3390/rs12101588.

Yasyukevich Yu., Mylnikova A., Vesnin A. GNSS-Based Non-Negative Absolute Ionosphere Total Electron Content, its Spatial Gradients, Time Derivatives and Differential Code Biases: Bounded-Variable Least-Squares and Taylor Series. Sensors. 2020b, vol. 20, 5702. DOI: 10.3390/s20195702.

URL: https://omniweb.gsfc.nasa.gov/vitmo/cgm.html (accessed May 1, 2021).

How to cite this article

Yasyukevich A.S. Features of short-period variability of total electron content at high and middle latitudes. Solar-Terrestrial Physics. 2021. Vol. 7. Iss. 4. P. 71-78. DOI: $10.12737 /$ stp-74202107. 